\title{
Escala de Bienestar Subjetivo, versión corta (EBS-8): Revalidación, invarianza de medición y teoría de respuesta al item
}

\author{
Nazira Calleja; Tere A. Mason; Olimpia Gómez Pérez
}

Cómo citar este artículo:

Calleja, N., Mason, T. A., \& Gómez Pérez, O. (2022). Escala de Bienestar Subjetivo, versión corta (EBS-8): Revalidación, invarianza de medición y teoría de respuesta al ítem. Acta Colombiana de Psicología, 25(1), 203-217.https://www.doi.org/10.14718/ ACP.2022.25.1.13

Recibido, septiembre 20/2020; Concepto de evaluación, agosto 12/2021; Aceptado, septiembre 28/2021

\author{
Nazira Calleja \\ ORCID: https://orcid.org/0000-0003-0131-8785 \\ Universidad Nacional Autónoma de México, Ciudad de México, México. \\ Tere A. Mason ${ }^{1}$ \\ ORCID: https://orcid.org/0000-0003-1183-8004 \\ Universidad Nacional Autónoma de México, Ciudad de México, México. \\ Olimpia Gómez Pérez \\ ORCID: https://orcid.org/0000-0001-7264-0398 \\ Universidad Anáhuac, Puebla, México.
}

\begin{abstract}
Resumen
La Escala de Bienestar Subjetivo (EBS-20) y su versión corta (EBS-8) fueron desarrolladas y validadas para medir en población hispanohablante la satisfacción con la vida y el afecto positivo. El objetivo del presente estudio fue obtener evidencias adicionales de validez para la EBS-8. Para ello, se aplicó la escala en tres estudios sucesivos a un total de 2259 mexicanos de 12 a 81 años. Los resultados del primer estudio permitieron confirmar la estructura unifactorial (CMIN/DF $=2.336$; $\mathrm{CFI}=.996 ;$ RMSEA $=.047)$ y la adecuada confiabilidad de la escala $(\alpha=.958, \omega=.957)$; en el segundo se probó su invarianza de medición por sexo, grupo social y edad $(\Delta \chi 2 p>.05, \Delta \mathrm{CFI}<.004, \Delta \mathrm{RMSEA}<.014)$; y en el tercer estudio se encontró, mediante la calibración de sus reactivos bajo la TRI, que la escala estima satisfactoriamente el constructo evaluado ( $\alpha>1.69$; $\beta_{1}$ entre -2.78 y -2.35). A partir de los índices psicométricos obtenidos, se concluye que la EBs- 8 es un excelente instrumento breve construido en español para evaluar el bienestar subjetivo en adolescentes, jóvenes, adultos y adultos mayores.

Palabras clave: bienestar subjetivo, validación, análisis psicométrico, invarianza factorial, teoría de respuesta al ítem.
\end{abstract}

1 Facultad de Psicología, UNAM, Av. Universidad 3004, Col. Copilco-Universidad, Coyoacán, C. P.: 04510, CDMX, México. terealmason@hotmail.com

Este artículo es parte de la línea de investigación "Mejores prácticas en la medición de constructos psicosociales y de la salud", de la Facultad de Psicología de la Universidad Nacional Autónoma de México. Agradecemos a nuestros estudiantes de licenciatura y posgrado su dedicado esfuerzo en la recolección de los datos. 


\title{
Subjective Well-Being Scale, short version (EBS-8): Revalidation, measurement invariance and Item Response Theory
}

\begin{abstract}
The Subjective Well-Being Scale (EBS-20) and its short version (EBS-8) were developed and validated to measure life satisfaction and positive affect in a Spanish-speaking population. The aim of the present study was to obtain additional evidence of validity for the EBS-8. To this end, the scale was applied in three successive studies to a total of 2259 Mexicans aged 12 to 81 years. The results of the first study allowed us to confirm its unifactorial structure (CMIN/DF $=2.336$; CFI $=.996$; RMSEA $=.047)$ and its adequate reliability $(\alpha=.958, \omega=.957)$; in the second its measurement invariance by sex, social group and age was tested $(\Delta \chi 2 \mathrm{p}>.05, \Delta \mathrm{CFI}<.004, \Delta \mathrm{RMSEA}<.014)$; and in the third study it was found, by calibrating its items under IRT, that the scale satisfactorily estimates the construct assessed ( $\mathrm{a}>1.69$; b1 between -2.78 and -2.35 ). From the psychometric indices obtained, it is concluded that the EBS-8 is an excellent brief instrument constructed in Spanish to assess subjective well-being in adolescents, young people, adults and older adults.

Keywords: subjective well-being, validation, psychometric analysis, factorial invariance, item response theory.
\end{abstract}

\section{Introducción}

El interés en el bienestar subjetivo (BS) ha tenido un gran crecimiento en las últimas décadas y ha generado contribuciones significativas en áreas tan diversas como la salud y las conductas saludables (Kushlev et al., 2020; Levine et al., 2021), el comportamiento organizacional (Hassan Butt et al., 2020), la crianza infantil (Temiz, 2020), la enseñanza-aprendizaje (Harding et al., 2019), entre otras. Una amplia revisión de investigaciones empíricas sobre las variables asociadas con el BS, principalmente aquellas relativas a valores culturales, puede encontrarse en Gutiérrez-Carmona y Urzúa (2019).

Específicamente, el BS se refiere a la creencia o sensación que tiene una persona de que su vida va bien (Diener et al., 2018), e incluye tanto evaluaciones cognitivas - la satisfacción con lo alcanzado en vida y respecto a las metas deseadas - como afectivas - las emociones positivas y negativas experimentadas en la vida cotidiana-(Cuadra \& Florenzano, 2003). No obstante, es importante señalar que el BS se diferencia del concepto de bienestar psicológico - eudaimónico-, el cual tiene que ver con el sentido de la vida, el crecimiento personal y las relaciones positivas, sin el componente afectivo (Keyes et al., 2002).

Por tanto, la medición del BS es clave en la investigación en este campo. En este sentido, numerosos estudios se han enfocado en desarrollar instrumentos, principalmente de autoinforme, y obtener sus propiedades psicométricas (Pavot, 2018). En general, se ha encontrado que la Satisfaction with Life Scale (swLS), de Diener et al. (1985), es la escala unifactorial más utilizada para medir los aspectos cognitivos del BS en jóvenes y adultos; la cual consta de solo cinco reactivos y siete opciones de respuesta.

La versión en español de la swLS se ha aplicado ampliamente en varios países hispanoamericanos (p. ej., Arias et al., 2018; Atienza et al., 2000; Gómez et al., 2007; Moyano Díaz \& Ramos Alvarado, 2007; Medrano et al., 2015; Padrós Blázquez et al., 2015; Vinaccia Alpi et al., 2019). Sin embargo, Casas et al. (2013) han reportado que, en algunas poblaciones, esta escala muestra un mal ajuste estadístico y, en particular, dificultades en uno de sus reactivos - "Si volviese a nacer, cambiaría bastantes cosas en mi vida" -; de hecho, Castellá Sarriera et al. (2012) decidieron retirar dicho reactivo de su estudio porque los adolescentes difícilmente lo comprendían.

Puesto que la SwLS solo evalúa la satisfacción con la vida, es común que se aplique con las Escalas de Afecto Positivo $y$ Afecto Negativo (PANAS), de Watson et al. (1988), para medir el componente afectivo. Este instrumento consta de una lista de 20 emociones, aunque no todas correspondientes a los afectos hedónicos a los que se hace referencia en la definición de BS. En las adaptaciones al español del PANAS, los investigadores han encontrado ambigüedades en la interpretación de los reactivos (Lapuente et al., 2018), por lo que han optado por eliminar adjetivos problemáticos (Flores-Kanter et al., 2021) o agregar la definición de cada uno con el fin de evitar las dificultades detectadas en su comprensión (Cantor Parra \& Clavijo Benavides, 2020). 
Teniendo esto en cuenta, recientemente, Calleja y Mason (2020) desarrollaron un instrumento para evaluar, en una sola escala, la satisfacción con la vida y el afecto positivo en población mexicana. Los reactivos fueron generados en grupos focales, por lo que su fraseo reflejó los modos de expresión de los participantes. Con los 20 reactivos -10 por dimensión- que obtuvieron puntajes más altos en validez de contenido evaluada por expertos, se conformó la EBS-20, que fue respondida por estudiantes universitarios en dos estudios sucesivos. Todos los análisis de discriminación de reactivos resultaron favorables. La estructura bidimensional de la EBS-20 fue probada satisfactoriamente con análisis factoriales exploratorios y confirmatorios; los índices de consistencia interna indicaron que la escala era altamente confiable $(\alpha=.970$ y $\omega=.958)$; e incluso se obtuvo evidencia de su validez de criterio con correlaciones estadísticamente significativas entre el puntaje de la EBS-20 y los del Cuestionario de Optimismo (Pedrosa et al., 2015), la Escala de Gratitud (McCullough et al., 2002), la Medida Analógica de Felicidad - versión adaptada de la Escalera de Cantril (1965)_, y la Escala de Soledad (Montero y López Lena, 1998).

A partir de la EBS-20, las autoras desarrollaron y validaron una versión corta con ocho reactivos - cuatro de satisfacción con la vida y cuatro de afecto positivo-, cuyo modelo unifactorial ajustó correctamente, y cuyos índices psicométricos fueron similares a los de la versión de 20 reactivos (Calleja \& Mason, 2020). En específico, la EBS-8 es un instrumento de aplicación breve y práctica, menos demandante para quien lo responde, y que requiriere poco tiempo para su procesamiento; sin embargo, las muestras de los estudios de validación estuvieron constituidas por estudiantes universitarios, por lo que resultaba imperativo obtener datos de población abierta y de un amplio rango de edad con el fin de probar la calidad psicométrica de la escala en su versión abreviada.

Además, se requería evaluar su invarianza por sexo y edad, pues la invarianza de medición, que implica probar la equivalencia del constructo medido en dos o más grupos independientes, tiene el objetivo de garantizar que las diferencias encontradas entre los grupos sean diferencias verdaderas en la cantidad del constructo y no diferencias relativas a la calidad de los reactivos (Cheung \& Rensvold, 2002). Con el fin de probar la invarianza de medición, la técnica psicométrica más frecuentemente utilizada es el análisis factorial confirmatorio de grupos múltiples (Chen et al., 2005), el cual consiste en ejecutar una serie de modelos de ecuaciones estructurales cada vez más restrictivos y probar si las diferencias entre ellos son significativas. En este sentido, hay cuatro niveles de invarianza - de configuración, métrica, escalar y estricta-, y al establecer sucesivamente que las cargas factoriales, los interceptos y las varianzas de los residuales sean equivalentes en un modelo factorial, es posible asegurar que las comparaciones que se realicen entre dos o más grupos respecto a la variable latente resulten válidas (Caycho, 2017; Van de Schoot et al., 2012).

Asimismo, es recomendable obtener evidencias de validez desde una aproximación psicométrica alternativa a la conceptualización y a los análisis de la teoría clásica de los test (TCT), en este caso, desde la teoría de respuesta al item (TRI). Este enfoque establece que la respuesta a un reactivo en particular está influida tanto por las características de quien lo contesta como por las del propio reactivo, así que sus procedimientos permiten obtener información sobre la persona, los reactivos y la escala en su conjunto (Furr, 2018). La premisa básica de la TRI es que la probabilidad de una respuesta está en función del rasgo latente verdadero del individuo - en este caso, BS - , que se representa con Theta $(\theta)$, la cual se ha estandarizado en términos de la distribución normal — con un rango de -3.00 a 3.00, donde 0.00 representa el puntaje promedio-(Baker, 2001). Los análisis de la TRI permiten obtener las propiedades de discriminación (alfa) y dificultad (beta) de cada reactivo, con lo que es posible detectar si están midiendo adecuadamente el constructo. El alfa refleja la fuerza con la que un reactivo está relacionado con el rasgo latente, por lo que puede considerarse aproximadamente equivalente a las cargas factoriales en тСт (Nima et al., 2020).

La presente investigación constituye la segunda fase de los estudios de validación de la EBS, cuya Fase 1 se expuso en Calleja y Mason (2020; véase Figura 1). En particular, sus objetivos son revalidar la estructura unifactorial de la EBS -8 con personas de un amplio rango de edad (Estudio 1); obtener evidencias de validez a través de la prueba de modelos de invarianza para la EBS-8 por sexo, grupo social y edad (Estudio 2); $y$, finalmente, aplicar los procedimientos de la TRI para evaluar el funcionamiento de los reactivos y la validez interna-estructural de la escala en su conjunto, de acuerdo con este enfoque psicométrico (Estudio 3). 
Figura 1.

Objetivos y muestras de los estudios de validación de la EBS

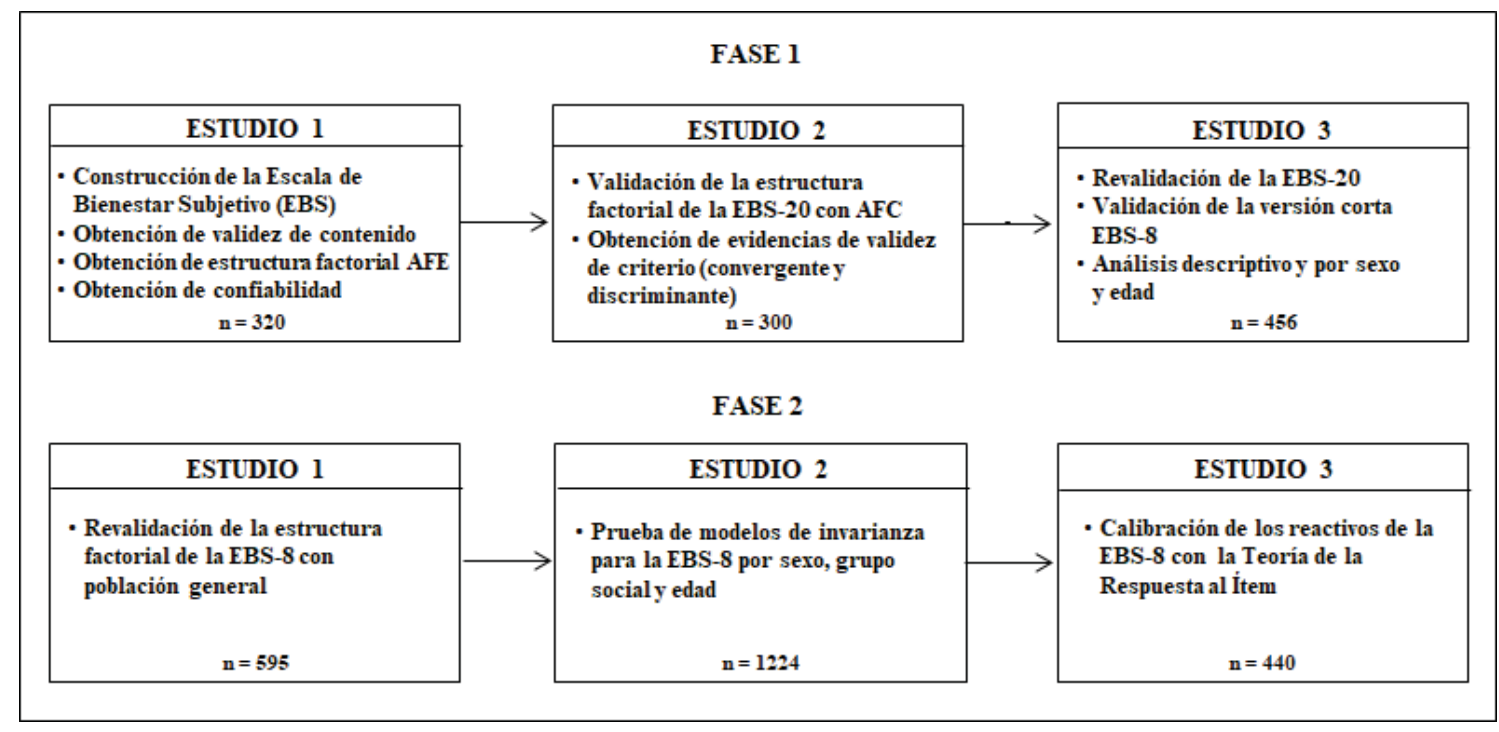

Nota. Los resultados de la Fase 1 se reportan en Calleja y Mason (2020), y los de la Fase 2 en el presente estudio.

\section{Estudio 1. Revalidación factorial de la EBS-8}

Método

Tipo de estudio y diseño

El tipo de investigación fue instrumental, ya que se analizaron las propiedades psicométricas de un instrumento de medición psicológica (Ato et al., 2013).

\section{Participantes}

Se conformó una muestra por conveniencia de 595 personas, habitantes de la Ciudad de México, de 18 a 81 años $(M=35.12, D E=12.57) ; 68.7 \%$ eran mujeres; y más de la mitad (57.8\%) dijeron ser solteros. El $41.5 \%$ de los participantes contaba con estudios de licenciatura, $27.2 \%$ de posgrado, y el resto $(31.3 \%)$, de preparatoria, de secundaria o de nivel técnico. El tamaño de la muestra de los tres estudios se determinó con base en la recomendación de Ferrando y Anguiano-Carrasco (2010), es decir, de un mínimo de 200 casos para evaluar la calidad psicométrica de un instrumento.

\section{Instrumento}

Se empleó la versión corta de la Escala de Bienestar Subjetivo (EBS-8), de Calleja y Mason (2020). Esta escala está integrada por ocho de los 20 reactivos que integran la EBS-20; cuatro de ellos corresponden a la dimensión de satisfacción con la vida — p. ej., "Me gusta mi vida"—, y cuatro a la de afecto positivo — p. ej., "Soy una persona feliz"- Los ocho reactivos fueron elegidos considerando tanto las correlaciones de cada reactivo con la escala total y con su factor (Stöber \& Joormann, 2001), como las cargas factoriales y las varianzas explicadas más altas. El modelo de un solo factor ajustó correctamente $\left(\chi_{(5)}^{2}=12.250 ; \mathrm{CMIN} /\right.$ $\mathrm{DF}=2.450 ; \mathrm{NFI}=.997 ; \mathrm{CFI}=.996 ; \mathrm{GFI}=.909 ; \mathrm{SRMR}=.007$; RMSEA $=.046$, IC $90 \%[.016, .097]), \operatorname{con} \alpha=.968$ y $\omega=.972$; las cargas factoriales y las $R^{2}$ obtenidas para cada uno de los ochos reactivos resultaron $>.800 \mathrm{y}>.700$, respectivamente. Se utilizaron seis opciones de respuesta no simétricas para disminuir el "efecto techo" que se presenta comúnmente en la medición del BS: 1 = "En desacuerdo", 2 = "Ni de acuerdo ni en desacuerdo", 3 = "Ligeramente de acuerdo", 4 = "De acuerdo", 5 = "Muy de acuerdo", 6 = "Totalmente de acuerdo".

\section{Procedimiento}

La escala y el cuestionario demográfico — en el que se registró edad, sexo, escolaridad y lugar de residencia- se elaboraron tanto en formato impreso como en versión electrónica, considerando que se ha reportado que las 
versiones electrónicas y las de papel son equivalentes en la medición de constructos psicológicos (Calleja et al., 2020; Determann et al., 2017). Los aplicadores de la versión impresa se presentaban en lugares públicos de la Ciudad de México, como parques, centros comerciales y salas de espera; explicaban al participante el propósito del estudio, el carácter completamente voluntario de su participación y el tratamiento anónimo y confidencial de sus respuestas; $\mathrm{y}$, finalmente, solicitaban el consentimiento informado. En las aplicaciones electrónicas este consentimiento se incluyó en la primera sección del instrumento. En este caso, se empleó la plataforma Google Forms y se efectuó la difusión mediante redes sociales digitales y por correo electrónico. No se otorgó ningún tipo de recompensa por la participación en el estudio.

Se tuvieron en cuenta los criterios éticos que rigen la investigación en psicología (Sociedad Mexicana de Psicología, 2010) en la realización de los estudios no intrusivos de la presente investigación.

\section{Análisis de los datos}

Inicialmente, para confirmar la estructura unifactorial de la EBS-8 se aplicó un análisis factorial confirmatorio ( $\mathrm{AFC})$, usando máxima verosimilitud como método de estimación. La normalidad multivariada de los datos se evaluó con la prueba de Mardia (Bollen, 1989). En este caso, los índices analizados para evaluar el ajuste del modelo fueron: la prueba de bondad de ajuste $\chi^{2}$, el cociente $\chi^{2} / g l(C M I N / D F)$, los índices comparativos CFI (Comparative Fit Index) y NFI (Normed Fit Index), los índices de proporción de varianza GFI (Goodness of Fit Index) y AGFI (Adjusted Goodness of Fit Index), y los índices de error SRMR (Standarized Root Mean Square Residual) y RMSEA (Root Mean Square of Aproximation) (Browne \& Cudeck, 1992). En la Figura 2 se indican los puntos de corte propuestos para cada uno de ellos (Hu \& Bentler, 1999). Por otra parte, se calculó la varianza media extraída (AVE) a partir de las $\lambda$ obtenidas. Para estos análisis se emplearon los programas SPSS y AMOS, ambos versión 22 .

\section{Resultados}

El coeficiente de curtosis multivariada obtenido con la prueba de Mardia resultó de 47.595, un valor inferior al límite indicado por Bollen (1989), que para ocho variables observadas sería: $8(8+2)=80$. El modelo resultante y los índices de ajuste para el modelo propuesto de un factor, obtenidos mediante el AFC, aparecen en la Figura 2. Todos ellos resultaron satisfactorios, al igual que los índices de consistencia interna $\alpha$ y $\omega$. Por otra parte, las cargas factoriales de los reactivos fueron significativas, la más baja correspondió al reactivo 8 - "Estoy "de buenas"”-, y el valor obtenido para la varianza media extraída $(A V E=.738)$ estuvo por encima del mínimo de .50, como lo recomiendan Fornell y Larcker (1981). Finalmente, la media de puntajes de los participantes fue de $4.52(D E=1.11)$, con rango de 1 a 6 .

\section{Estudio 2. Invarianza de medición}

\section{Método}

Tipo de estudio y diseño

El tipo de investigación fue instrumental, ya que se analizaron las propiedades psicométricas de un instrumento de medición psicológica (Ato et al., 2013).

\section{Participantes}

Los 1224 participantes de este estudio, independientes de quienes participaron en el Estudio 1, fueron agrupados en cuatro muestras distintas: adolescentes de secundaria, estudiantes de preparatoria, jóvenes universitarios, y adultos de la Ciudad de México. Su edad y su distribución por sexo se presentan en la Tabla 1. En general, se observó una proporción ligeramente mayor de mujeres que de hombres en todas las muestras, diferencia que no fue significativa $\left(\chi_{(3)}^{2}=5.152, p=.161\right)$. La edad de los estudiantes correspondió a su ciclo escolar -12 a 15 años para secundaria, 15 a 18 para preparatoria y 18 a 25 para universidad-, mientras que en los adultos no escolarizados la edad cubrió de los 18 años hasta la séptima década de la vida, y estos fueron agrupados de la siguiente manera: de 20 años o menos $(22.0 \%)$, de 21 a 30 años (33.1\%), de 31 a 40 años (35.6\%) y de más de 40 años $(9.3 \%)$ - la escolaridad de esta muestra iba de secundaria a estudios de posgrado-. 
Figura 2.

AFC de la EBS-8, sus índices de ajuste y de consistencia interna

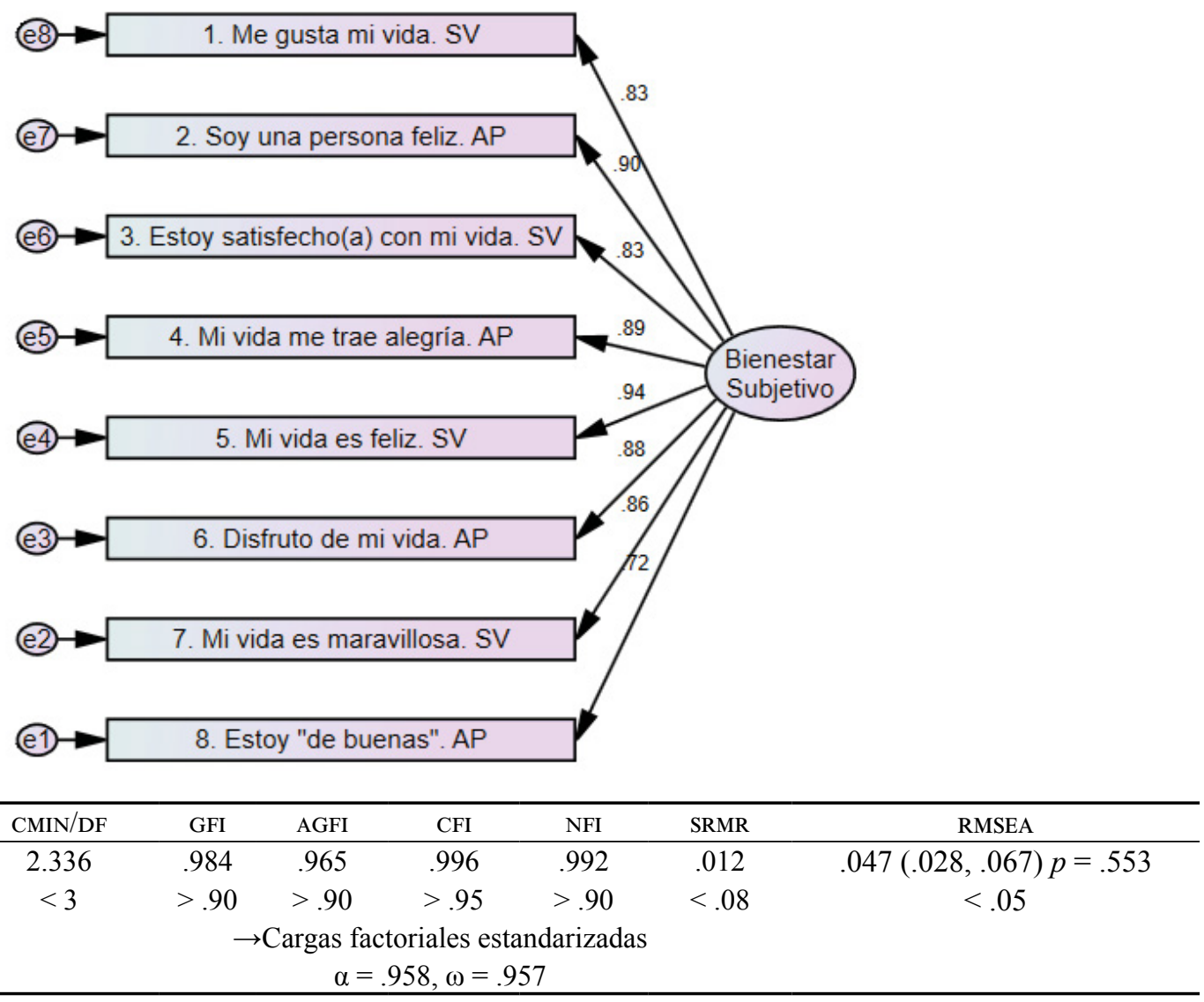

Nota. *Tomado de Hu y Bentler (1999).

Tabla 1.

Medidas descriptivas de la edad y distribución porcentual por sexo de las cuatro muestras de participantes del Estudio 2

\begin{tabular}{|c|c|c|c|c|c|}
\hline \multirow{2}{*}{ Muestra } & \multirow{2}{*}{$\mathrm{N}$} & \multicolumn{2}{|c|}{ Sexo } & \multicolumn{2}{|c|}{ Edad } \\
\hline & & Mujeres (\%) & Hombres (\%) & $M(D E)$ & Rango \\
\hline Adolescentes de secundaria & $292(23.9 \%)$ & 51.7 & 48.3 & $13.30(0.88)$ & $12-15$ \\
\hline Adolescentes de preparatoria & $356(29.1 \%)$ & 54.5 & 45.5 & $16.76(0.99)$ & $15-18$ \\
\hline Jóvenes universitarios & $326(26.6 \%)$ & 55.5 & 44.5 & $20.37(1.62)$ & $18-25$ \\
\hline Adultos de población general & $250(20.4 \%)$ & 66.8 & 37.2 & $29.79(10.85)$ & $18-69$ \\
\hline Total & $1224(100.0 \%)$ & 55.8 & 44.2 & $19.56(7.64)$ & $12-69$ \\
\hline
\end{tabular}

\section{Instrumento}

Se aplicó la EBs-8, cuya estructura factorial se confirmó en el Estudio 1, con seis opciones de respuesta — de 1 = "En desacuerdo" a 6 = "Totalmente de acuerdo"-.

\section{Procedimiento}

En el caso de las muestras de participantes menores de edad — estudiantes de secundaria y de preparatoria—, se solicitó la autorización de los directivos de los respectivos planteles y se requirió el consentimiento informado de los padres de familia. Para los participantes universitarios y de población general adulta se incluyó el consentimiento informado al inicio del instrumento. La aplicación a las muestras escolarizadas se efectuó en los salones de clase, y en población general se realizó en lugares públicos, como parques, centros comerciales y salas de espera. A todos se 
les pedía participar voluntaria y anónimamente en el estudio, se les informaba el objetivo del estudio, y se les aseguraba el tratamiento confidencial de los datos.

\section{Análisis de los datos}

Para evaluar la invarianza de medición - de configuración, métrica, escalar y estricta- por sexo, grupo social y edad, se efectuaron AFC multi-grupo (Vandenberg \& Lance, 2000), utilizando el programa AMOs, versión 22. La invarianza de configuración se refiere a la no variabilidad de la estructura factorial entre los grupos mientras se calculan libremente las cargas factoriales, los interceptos y los residuales; en la invarianza métrica se restringen la estructura factorial y las cargas factoriales; en la invarianza escalar se agrega la restricción de los interceptos; y en la invarianza estricta se encuentran restringidos, además de los dos índices anteriores, los residuales. Para evaluar las diferencias de los modelos, se utilizaron como índices los cambios en CFI y en RMSEA, y la significancia del $\Delta \chi^{2}$. Se apoya una fuerte invarianza cuando el $\Delta$ CFI $\leq .01$, el $\Delta$ RMSEA $\leq .015$, y el $\Delta \chi^{2}, p>.05$ (Cheung \& Rensvold, 2002).

\section{Resultados}

Inicialmente, se efectuó un AFC multi-grupo para probar el modelo de invarianza de configuración, línea base o libre (M1), que proponía que la EBs- 8 tendría una estructura unifactorial en todos los grupos, y se permitió que las cargas factoriales, los interceptos y las varianzas de error se estimaran libremente. Como se observa en la Tabla 2, tanto en la desagregación por sexo como por grupo social y edad, todas las cargas factoriales fueron altas y significativas $(p<.001)$. En general, las cargas más altas correspondieron al reactivo 5 , y las más bajas, al 8. Los índices obtenidos ( $\mathrm{CFI}=.996$; RMSEA $=.038$; $\left.\chi^{2} / g l=2.746\right)$ indicaron que el ajuste del modelo a los datos era excelente.

Por otra parte, en la Tabla 3 se muestran los resultados de las pruebas de los modelos de invarianza métrica o débil (M2), de invarianza escalar o fuerte (M3), y de invarianza estricta (M4). En general, las puntuaciones indicaron que cuando las cargas factoriales, interceptos y residuales de la estructura factorial se mantuvieron progresivamente invariantes en función del sexo y del grupo social, los índices de ajuste fueron comparables, por lo que la medición del BS con la EBS- 8 de hombres y mujeres, y de estudiantes de secundaria, preparatoria y universidad, así como de personas adultas de la población general, no varía. Por tanto, los puntajes podrían ser comparados entre los grupos, y el cambio en una unidad sería equivalente entre ellos. En relación con la invarianza a través de la edad del grupo de población general, los criterios de $\Delta$ CFI e $\Delta$ RMSEA fueron alcanzados en los modelos 2, 3 y 4; sin embargo, el $\Delta \chi^{2}$ resultó no significativo para los modelos 2 y 3 , pero no para el 4, por lo que faltó este último parámetro de la invarianza estricta para obtener la equivalencia completa de la estructura de la escala por edad.

Tabla 2.

Cargas factoriales estandarizadas en los AFC multi-grupo del modelo de invarianza de configuración

\begin{tabular}{|c|c|c|c|c|c|c|c|c|c|c|}
\hline \multirow{2}{*}{ Reactivo } & \multicolumn{2}{|c|}{$\begin{array}{c}\text { Cargas factoriales } \\
\text { por sexo }\end{array}$} & \multicolumn{4}{|c|}{ Cargas factoriales por grupo social } & \multicolumn{4}{|c|}{$\begin{array}{c}\text { Cargas factoriales por edad } \\
\text { (población general) }\end{array}$} \\
\hline & Mujeres & Hombres & Secundaria & Preparatoria & Universidad & Pob. gral. & $\begin{array}{c}200 \\
\text { menos }\end{array}$ & 21 a 30 & 31 a 40 & $\begin{array}{l}\text { Más de } \\
40\end{array}$ \\
\hline 1. Me gusta mi vida. & $.909 *$ & $.863 *$ & $.809 *$ & $.895^{*}$ & $.908^{*}$ & $.900 *$ & $.946^{*}$ & $.949 *$ & $.870 *$ & $.813^{*}$ \\
\hline 2. Soy una persona feliz. & $.876^{*}$ & $.862 *$ & $.836^{*}$ & $.873 *$ & $.911 *$ & $.879 *$ & $.792 *$ & $.839 *$ & $.964 *$ & $.973 *$ \\
\hline $\begin{array}{l}\text { 3. Estoy satisfecho(a) con } \\
\text { mi vida. }\end{array}$ & $.873 *$ & $.882 *$ & $.819 *$ & $.912 *$ & $.878^{*}$ & $.881 *$ & $.850 *$ & $.838^{*}$ & $.937 *$ & $.768^{*}$ \\
\hline 4. Mi vida me trae alegría. & $.917^{*}$ & $.903 *$ & $.898 *$ & $.909 *$ & $.931 *$ & $.934 *$ & $.908^{*}$ & $.927 *$ & $.972 *$ & $.979 *$ \\
\hline 5. Mi vida es feliz. & $.937 *$ & $.921 *$ & $.888 *$ & $.921 *$ & $.935^{*}$ & $.936^{*}$ & $.855^{*}$ & $.957 *$ & $.962 *$ & $.968^{*}$ \\
\hline 6. Disfruto de mi vida. & $.887 *$ & $.887 *$ & $.877 *$ & $.907 *$ & $.903 *$ & $.904 *$ & $.912 *$ & $.840^{*}$ & $.932 *$ & $.853^{*}$ \\
\hline 7. Mi vida es maravillosa. & $.866^{*}$ & $.834 *$ & $.846^{*}$ & $.875^{*}$ & $.845^{*}$ & $.885^{*}$ & $.818^{*}$ & $.851 *$ & $.937 *$ & $.718^{*}$ \\
\hline 8. Estoy "de buenas". & $.736^{*}$ & $.673 *$ & $.712 *$ & $.685^{*}$ & $.749 *$ & $.758 *$ & $.775 *$ & $.684^{*}$ & $.857 *$ & $.673^{*}$ \\
\hline
\end{tabular}

Nota. $* p<.001$. 
Tabla 3.

Índices de ajuste para las pruebas de los modelos de invarianza por sexo, por grupo social y por edad

\begin{tabular}{|c|c|c|c|c|c|c|c|c|}
\hline Modelo & $\chi^{2}(g l)$ & $\chi^{2} / g l$ & CFI & $\begin{array}{c}\text { RMSEA } \\
(\text { IC } 90 \%)\end{array}$ & Comparación & $\Delta \chi^{2}$ & $\Delta \mathrm{CFI}$ & $\triangle$ RMSEA \\
\hline \multicolumn{9}{|l|}{ Modelos de invarianza por sexo } \\
\hline $\begin{array}{l}\text { M1. Invarianza de } \\
\text { configuración } \\
\text { (línea base) }\end{array}$ & $60.418(22)$ & 2.746 & .996 & $\begin{array}{c}.038 \\
(.027-.049)\end{array}$ & & & & \\
\hline $\begin{array}{l}\text { M2. Invarianza métrica o débil } \\
(\lambda \text { restringidas })\end{array}$ & $64.508(29)$ & 2.224 & .997 & $\begin{array}{c}.032 \\
(.021-.042)\end{array}$ & M2 vs. M1 & $\begin{array}{l}4.090(7) \\
p=.769\end{array}$ & .001 & .006 \\
\hline $\begin{array}{l}\text { M3. Invarianza escalar o fuerte } \\
(\lambda \text { y } \tau \text { restringidos })\end{array}$ & $75.415(37)$ & 2.309 & .995 & $\begin{array}{c}.033 \\
(.024-.042)\end{array}$ & M3 vs. M2 & $\begin{array}{c}10.907(8) \\
p=.207\end{array}$ & .002 & -.001 \\
\hline $\begin{array}{l}\text { M4. Invarianza estricta } \\
(\lambda, \tau, \theta \text { y restringidos })\end{array}$ & $106.132(57)$ & 2.293 & .993 & $\begin{array}{c}.033 \\
(.025-.040)\end{array}$ & M4 vs. M3 & $\begin{array}{c}30.717(20) \\
p=.059\end{array}$ & -.002 & .000 \\
\hline \multicolumn{9}{|c|}{ Modelos de invarianza por grupo social } \\
\hline $\begin{array}{l}\text { M1. Invarianza de } \\
\text { configuración } \\
\text { (línea base) }\end{array}$ & $97.957(32)$ & 3.061 & .982 & $\begin{array}{c}.040 \\
(.034-.045)\end{array}$ & & & & \\
\hline $\begin{array}{l}\text { M2. Invarianza métrica o débil } \\
(\lambda \text { restringidas })\end{array}$ & $114.614(42)$ & 2.728 & .979 & $\begin{array}{c}.037 \\
(.032-.042)\end{array}$ & M2 vs. M1 & $\begin{array}{c}16.657(10) \\
p=.082\end{array}$ & .003 & .003 \\
\hline $\begin{array}{l}\text { M3. Invarianza escalar o fuerte } \\
(\lambda \text { y } \tau \text { restringidos })\end{array}$ & $139.660(58)$ & 2.407 & .975 & $\begin{array}{c}.036 \\
(.032-.041)\end{array}$ & M3 vs. M2 & $\begin{array}{c}25.045(16) \\
p=.069\end{array}$ & .004 & .001 \\
\hline $\begin{array}{l}\text { M4. Invarianza estricta } \\
(\lambda, \tau, \theta \text { y restringidos })\end{array}$ & $144.21(54)$ & 2.670 & .974 & $\begin{array}{c}.037 \\
(.032-.041)\end{array}$ & M4 vs. M3 & $\begin{array}{l}4.550(4) \\
p=.336\end{array}$ & .001 & -.001 \\
\hline \multicolumn{9}{|c|}{ Modelos de invarianza por edad (población general) } \\
\hline $\begin{array}{l}\text { M1. Invarianza de } \\
\text { configuración } \\
\text { (línea base) }\end{array}$ & $117.982(64)$ & 1.843 & .955 & $\begin{array}{c}.076 \\
(.071-.120)\end{array}$ & & & & \\
\hline $\begin{array}{l}\text { M2. Invarianza métrica o débil } \\
(\lambda \text { restringidas })\end{array}$ & $136.659(85)$ & 1.608 & .957 & $\begin{array}{c}.073 \\
(.049-.095)\end{array}$ & M2 vs. M1 & $\begin{array}{l}18.677(21) \\
p=.606\end{array}$ & .002 & -.003 \\
\hline $\begin{array}{l}\text { M3. Invarianza escalar o fuerte } \\
\text { ( } \lambda \text { y } \tau \text { restringidos })\end{array}$ & $162.750(109)$ & 1.493 & .955 & $\begin{array}{c}.066 \\
(.043-.086)\end{array}$ & M3 vs. M2 & $\begin{array}{c}26.091(24) \\
p=.349\end{array}$ & .004 & -.007 \\
\hline $\begin{array}{l}\text { M4. Invarianza estricta } \\
(\lambda, \tau, \theta \text { y restringidos })\end{array}$ & $255.718(148)$ & 1.728 & 910 & $\begin{array}{c}.080 \\
(.063-.096)\end{array}$ & M4 vs. M3 & $\begin{array}{c}92.968(39) \\
p<.001\end{array}$ & .001 & .014 \\
\hline Criterios de corte & & & & & & $p>.05$ & $\leq .01$ & $\leq .015$ \\
\hline
\end{tabular}

Nota. $\lambda$ : cargas factoriales; $\tau$ : interceptos; $\theta$ : varianzas de error; CFI: índice de ajuste comparativo; RMSEA: raíz del error cuadrático medio.

\section{Estudio 3. Calibración de la EBS-8 con TRI}

Método

Tipo de estudio y diseño

El tipo de investigación fue instrumental, ya que se analizaron las propiedades psicométricas de un instrumento de medición psicológica (Ato et al., 2013).

\section{Participantes}

La muestra estuvo constituida por 440 estudiantes universitarios de licenciatura, hombres $(42.2 \%)$ y mujeres $(57.8 \%)$, de 18 a 25 años $(M=20.37, D E=1.75)$, habitantes de la Ciudad de México. Esta muestra fue independiente de las de los estudios 1 y 2 .

\section{Instrumento}

Se aplicó la EBS-8, con seis opciones de respuesta — de $1=$ "En desacuerdo" a 6 = "Totalmente de acuerdo"-, cuya estructura factorial se confirmó en el Estudio $1 \mathrm{y}$ cuya invarianza de medición fue probada en el Estudio 2.

\section{Procedimiento}

La escala fue aplicada en formato impreso - en los campus universitarios y en los salones de clase de los estudiantes - y en línea - en Google Forms, E-survey 
Creator y Adobe Acrobat IX-. El consentimiento informado, las instrucciones y las especificaciones respecto a la participación voluntaria y anónima y la confidencialidad de las respuestas fueron los mismos que los señalados en los estudios anteriores.

\section{Análisis de los datos}

Dentro del marco de la TRI, se estimaron los parámetros de discriminación (alfa, $\alpha$ ) y dificultad (beta, $\beta$ ) de los ocho reactivos de la versión corta, bajo el modelo de respuesta graduada de Samejima (1969). De acuerdo con Baker (2001), el valor del parámetro $\alpha$ de .65 se utiliza como un umbral mínimo de un reactivo que tiene un funcionamiento aceptable; un $\alpha>1.34$ indica que el funcionamiento del reactivo es alto; y un $\alpha>1.69$, muy alto. El parámetro de dificultad o localización $\beta$ debe oscilar entre $-3 \mathrm{y}+3$, sin traslaparse. Se estimó la información aportada por cada uno de los reactivos y por la escala total en un rango de theta $\theta= \pm 3$. Los análisis se efectuaron utilizando el programa IRTPRO, versión 4.1 .

\section{Resultados}

Se estimaron los parámetros de discriminación y dificultad para cada uno de los reactivos de la EBS-8 (véase Tabla 4). En general, el poder discriminativo de todos ellos fue muy alto $(\alpha>1.69)$, siendo el más discriminativo el ítem 4 - "Mi vida me trae alegría"-, y el menos discriminativo, el ítem 8 - "Estoy 'de buenas" - En cuanto a su dificultad, los parámetros $\beta_{1}$ se ubicaron entre -2.78 (ítem 5 - "Mi vida es feliz"-) y-2.35 (item 6 - Disfruto de mi vida"-); para todos los reactivos, los valores para las alternativas de respuesta restantes $\left(\beta_{2}, \beta_{3}, \beta_{4}, \beta_{5}\right.$ y $\left.\beta_{6}\right)$ fueron decrecientes. Respecto a la prueba de ajuste de los reactivos al modelo, para dos de ellos (el ítem 8 - "Estoy 'de buenas" -, y el ítem 6 - "Disfruto de mi vida"-) el valor de $\chi^{2}$ resultó estadísticamente significativo $(<.01)$, lo cual, considerando el ajuste por los múltiples contrastes estadísticos, no parece afectar su comportamiento. Respecto a la función de información de los reactivos y de la escala total, se observó más información en los niveles bajos del constructo, ya que ninguno de los reactivos maximizó la información en los niveles muy elevados de Bs, lo cual implica que el error de medición aumenta en estos niveles (véase Figura 3). Las curvas características de cada uno de los ocho reactivos se muestran en la Figura 4.

\section{Discusión}

El propósito de la presente investigación fue obtener, mediante tres estudios sucesivos, evidencias adicionales de validez y confiabilidad para la EBS- 8 , versión corta de la EBS-20, desarrollada para medir el bienestar subjetivo (BS) en población hispanohablante. En particular, esta escala evalúa la satisfacción con la vida y el afecto positivo, dos de los tres componentes que, según se ha señalado (Diener et al., 2018), conforman el constructo de BS. Como se estipuló en Calleja y Mason (2020), el tercer componente - el afecto negativo - no se incluyó en la EBS debido a que la evidencia que apoya la ortogonalidad de ambos afectos es controversial (Flores-Kanter \& Medrano, 2018; Moral de la Rubia, 2011); además, según Diener et al. (2018),

Tabla 4.

Parámetros estimados según el modelo de respuesta graduada para los reactivos de la EBS-8

\begin{tabular}{cccccccccccc}
\hline Reactivo & $\alpha$ & $\beta_{1}$ & $\beta_{2}$ & $\beta_{3}$ & $\beta_{4}$ & $\beta_{5}$ & $\beta_{6}$ & $\chi^{2}$ & $g l$ & $p$ \\
\hline 1 & 3.76 & -2.60 & -2.11 & -1.53 & -0.69 & -0.24 & 0.40 & 81.09 & 62 & .055 \\
2 & 5.05 & -2.43 & -1.99 & -1.33 & -0.63 & -0.13 & 0.58 & 66.57 & 51 & .070 \\
3 & 3.61 & -2.44 & -1.93 & -1.24 & -0.56 & -0.04 & 0.73 & 70.42 & 65 & .300 \\
4 & 6.14 & -2.57 & -1.71 & -1.29 & -0.49 & -0.10 & 0.49 & 35.88 & 46 & .858 \\
5 & 3.51 & -2.78 & -1.98 & -1.38 & -0.58 & -0.06 & 0.65 & 72.35 & 70 & .399 \\
6 & 6.08 & -2.35 & -1.88 & -1.23 & -0.59 & -0.20 & 0.37 & 80.96 & 47 & $<.01$ \\
7 & 4.12 & -2.50 & -1.75 & -1.08 & -0.35 & 0.02 & 0.64 & 76.63 & 63 & .116 \\
8 & 3.39 & -2.53 & -1.99 & -1.33 & -0.63 & -0.10 & 0.68 & 107.05 & 68 & $<.01$ \\
\hline
\end{tabular}

Nota. $\alpha$ : parámetro de discriminación $-\alpha>1.69$ indica funcionamiento muy alto-; $\beta_{1}-\beta_{6}$ : valores de dificultad o localización que reflejan el cambio de una categoría de respuesta a otra - debe oscilar entre $-3 \mathrm{y}+3$, sin traslaparse- 
Figura 3.

Función de información de la EBS-8

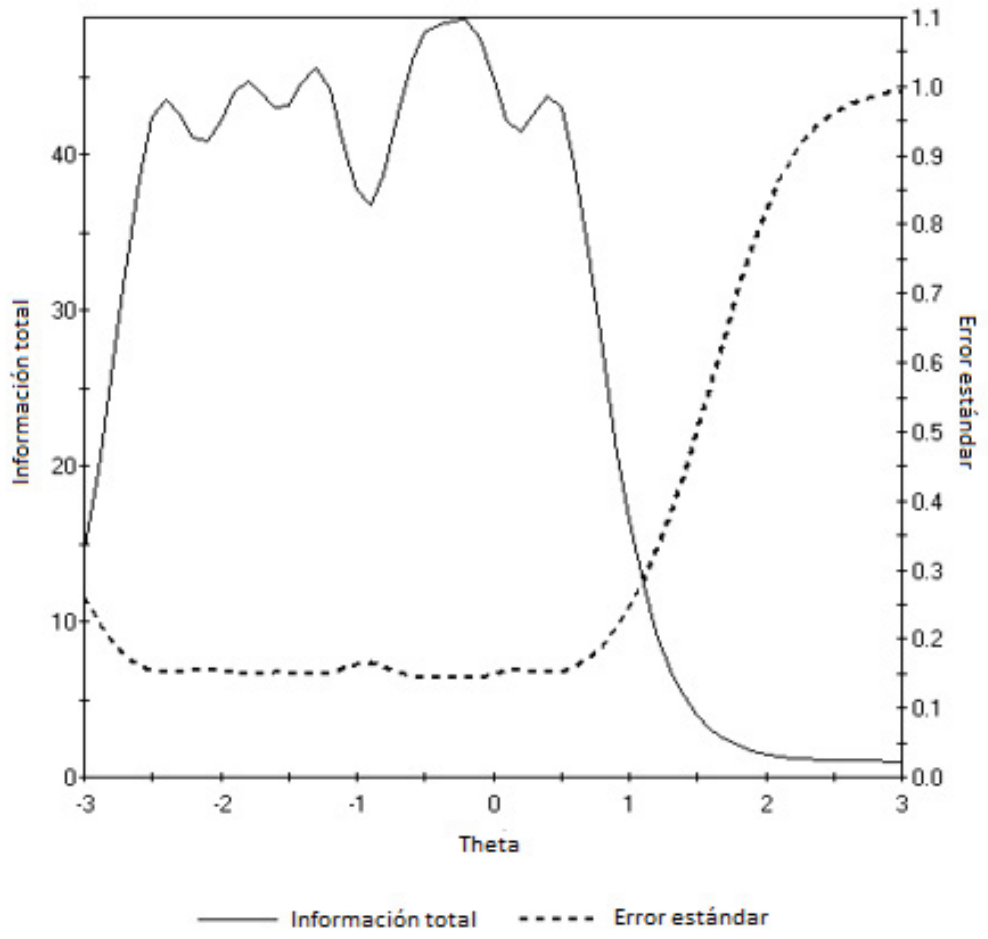

Figura 4.

Curvas características de las categorías de respuesta de los reactivos de la EBS-8

1. Me gusta mi vida

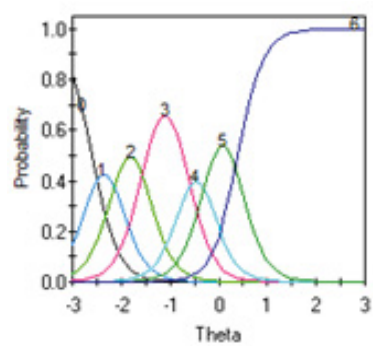

5. Mi vida es feliz

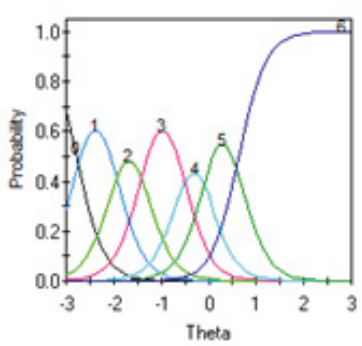

2. Soy una persona feliz

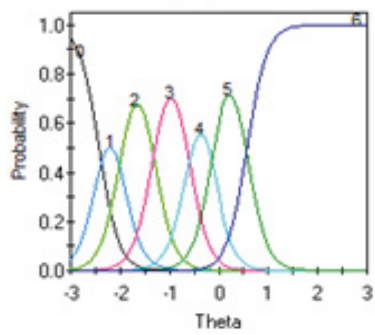

6. Disfruto de mi vida

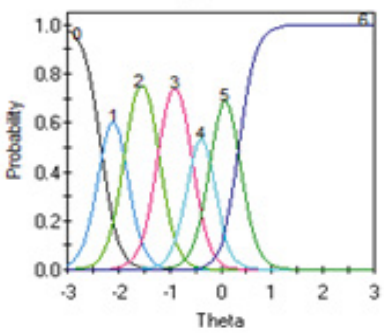

3. Estoy satisfecho (a) con mi vida

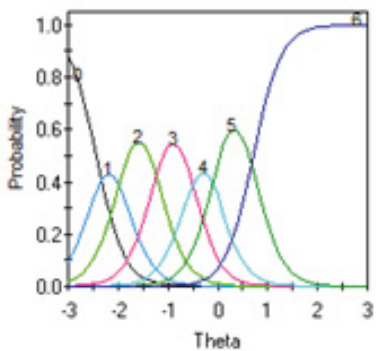

7. Mi vida es maravillosa

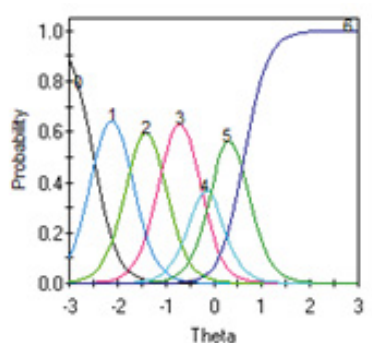

4. Mi vida me trae alegría

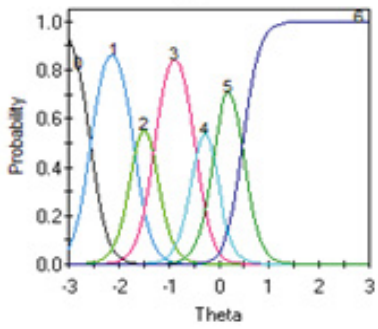

8. Estoy "de buenas"

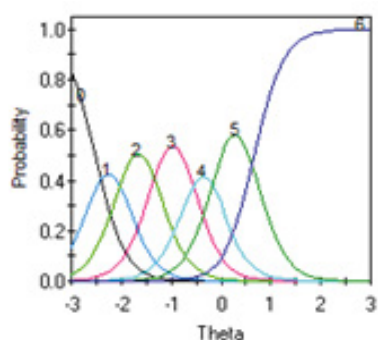


las personas que se consideran satisfechas con su vida tienden a experimentar emociones positivas.

Los índices del AFC obtenidos para la EBS-8 unifactorial en el Estudio 1 mostraron nuevamente el ajuste del modelo a los datos, como lo hicieron en Calleja y Mason (2020), pero esta vez con una muestra de población general con edades que oscilan entre los 18 y los 81 años. De acuerdo con esto, se puede afirmar que los datos apoyan la generalización de la validez de estructura de la EBS-8.

$\mathrm{Al}$ analizar - en el Estudio 2- la invarianza factorial de la EBS-8, es decir, evaluar el grado de igualdad o equivalencia de la estructura de la escala, se encontró que, de acuerdo con los criterios recomendados por Cheung y Rensvold (2002), los cuatro modelos puestos a prueba eran básicamente comparables cuando los reactivos sucesivos de la conformación factorial - cargas factoriales, interceptos y residuales - se mantenían invariantes independientemente del sexo. Los resultados descritos apoyan los hallazgos de estudios que han probado la invarianza escalar o estricta por sexo de escalas de Bs (p. ej. Checa et al., 2019; Moksnes et al., 2014; Tomás et al., 2015), lo cual significa que las comparaciones entre hombres y mujeres resultan apropiadas. Además, las evidencias de invarianza escalar entre estudiantes de diferentes niveles escolares y de personas de la población general sugieren que es posible realizar comparaciones válidas de los puntajes de Bs entre estos grupos sociales.

Respecto a la invarianza a través de la edad, las pruebas de invarianza efectuadas en el grupo de población general mostraron equivalencia de la estructura factorial entre personas de 20 años o menos, de 21 a 30, de 30 a 40 y de más de 40 años, excepto en uno de los parámetros del modelo de invarianza estricta; en este caso, se asumiría una invarianza parcial (Dimitrov, 2010), si bien se ha reconocido que las pruebas de invarianza estricta son excesivamente restrictivas (Bentler, 2004).

Por otra parte, los resultados del análisis de la TRI, bajo el modelo de respuesta graduada de Samejima (1969) - efectuados en el Estudio 3- indicaron que el constructo de BS se estima de manera aceptable con la forma corta de la EBS. Considerando los criterios establecidos (Baker, 2001), los parámetros alfa indicaron que los reactivos tuvieron alta capacidad discriminativa. Adicionalmente, los errores de estimación de los parámetros $\beta_{1}$ de todos los reactivos fueron superiores a los de los otros valores, lo cual posiblemente pueda ser explicado por la asimetría negativa de la distribución de los puntajes, ya que las opciones de respuesta que denotan menor Bs fueron poco elegidas por los participantes. Asimismo, se encontró que los reactivos no discriminaron bien en los niveles elevados del constructo $\left(\beta_{5}, \beta_{6}\right.$-alternativas 5 y $\left.6-\right)$, lo cual quiere decir que el error de medición aumenta cuando se pretende discriminar a los individuos con alto BS. Aun así, la confiabilidad de la escala fue elevada.

Respecto del análisis específico del reactivo 8 - "Estoy 'de buenas"'-, si bien sus valores se encuentran dentro de lo esperado, resultó el reactivo comparativamente menos discriminativo. Posiblemente resulte conveniente modificar su fraseo de "Estoy 'de buenas" a "Me siento 'de buenas", será necesario observar su desempeño en aplicaciones futuras de la escala.

En general, los resultados de los tres estudios de las propiedades psicométricas de la versión corta de la Escala de Bienestar Subjetivo (EBS-8), con un factor y ocho reactivos, en los que participaron en total 2259 personas de 12 a 81 años, mostraron evidencias de validez de estructura factorial, de invarianza por sexo, grupo social y edad, y de confiabilidad. De este modo, además de las evidencias obtenidas bajo el enfoque de la тст, la calibración de los reactivos con metodología de la TRI fue satisfactoria. Aun cuando el uso de la TRI en la validación de instrumentos psicosociales es poco frecuente, sus herramientas de análisis de reactivos proporcionan rigurosidad y objetividad a la medición de constructos psicológicos (Simms, 2008), como se mostró en esta investigación.

Por lo tanto, dados los excelentes índices de confiabilidad y validez que posee la EBS-8, y por ser un instrumento parsimonioso por su brevedad, esta escala puede equipararse con otras que tienen actualmente una amplia utilización en México y en otros países hispanohablantes, como ocurre con las traducciones-adaptaciones de la SwLs (Diener et al., 1985), que evalúa la dimensión cognitiva-valorativa, y del PANAS (Watson et al., 1988), que mide la dimensión afectivo-emocional. Tanto la SWLS como el PANAS han sido desarrollados con participantes - mayormente estudiantes universitarios - de países anglosajones, de tal manera que, aún adaptados, presentan reactivos con interpretaciones diferentes en nuestras culturas. De hecho, para la SwLS, 
de cinco reactivos, se han reportado índices de confiabilidad menores que los obtenidos para la EBs-8 (Atienza et al., 2000, $\alpha=.86$; Gómez et al., 2007, $\alpha=.84$; Moyano Díaz $\&$ Ramos Alvarado, 2007, $\alpha=.87$; Padrós Blázquez et al., $2015, \alpha=.83$ ); y del PANAS, con 20 reactivos, habría que decir que no fue creado expresamente para evaluar el Bs, que usa un formato distinto - lista de emociones - y que los estados de ánimo positivo de algunos de sus reactivos - - . ej., alerta, atento, inspirado, fuerte, decidido o excitadono son las emociones o afectos positivos - hedónicas - a los que se hace referencia en la definición de BS.

Finalmente, si bien en esta investigación se han obtenido excelentes índices psicométricos para la EBS- 8 en adolescentes, jóvenes, adultos y adultos mayores mexicanos, su utilidad en ámbitos sociales, clínicos y laborales e incluso en distintas regiones del país y de otras naciones de habla hispana tendrá que ser analizada con el fin de generalizar su validez. Otra limitante de la investigación fue no haber recabado un mayor número de variables sociodemográficas de las muestras - como nivel socioeconómico y ocupación-, con el fin de que pudiera valorarse su aplicación en otras poblaciones hispanohablantes. Asimismo, aun cuando se reportaron evidencias de validez de criterio para la EBS-20 (Calleja \& Mason, 2020), con correlaciones significativas con constructos de su red nomológica — optimismo, gratitud, felicidad y soledad-, será necesario obtenerlas también para la EBS-8.

En conclusión, la EBS- 8 constituye un instrumento breve, válido y confiable para la medición del Bsen personas hispanohablantes, que puede usarse en aplicaciones intensivas, y que permite confirmar que el desarrollo y validación de escalas en nuestros países, como alternativa a las traducidas y adaptadas de otras culturas, también contribuye a la psicometría.

\section{Referencias}

Arias, W., Huamani, J., \& Caycho-Rodríguez, T. (2018). Satisfaction with life in high school students from the city of Arequipa. Propósitos y Representaciones, 6(1), 351-407. https://doi.org/10.20511/pyr2018.v6n1.206

Atienza, F. L., Pons, D., Balaguer, I., \& García-Merita, M. (2000). Propiedades psicométricas de la Escala de Satisfacción con la Vida en adolescentes. Psicothema, 12(2), 314-319. http://www.psicothema.com/pdf/296.pdf
Ato, M., López, J. J., \& Benavente, A. (2013). Un sistema de clasificación de los diseños de investigación en psicología. Anales de Psicología, 29(3), 1038-1059. https://doi. org/10.6018/analesps.29.3.178511

Baker, F. B. (2001). The basics of Item Response Theory. ERIC Clearinghouse on Assessment and Evaluation. https:// files.eric.ed.gov/fulltext/ED458219.pdf

Bentler, P. M. (2004). EQS 6: Structural equation program manual. Multivariate Software.

Bollen, K. A. (1989). Structural equations with latent variables. John Wiley \& Sons.

Browne, M. W., \& Cudeck, R. (1992). Alternative ways of assessing model fit. Sociological Methods \& Research, 21(2), 230-258. https://doi. org/10.1177/0049124192021002005

Calleja, N., Candelario Mosco, B. J., Rosas Medina, J. H., \& Souza Colín, E. (2020). Equivalencia psicométrica de las aplicaciones impresas y electrónicas de tres escalas psicosociales. Revista Argentina de Ciencias del Comportamiento, 12(2), 50-58. https://doi. org/10.32348/1852.4206.v12.n2.25284

Calleja, N., \& Mason, T. A. (2020). Escala de Bienestar Subjetivo (EBS-20 y EBS-8): Construcción y validación. Revista Iberoamericana de Diagnóstico y Evaluación Psicológica e Avaliação Psicológica, RIDEP, 55(2), 185201. https://doi.org/10.21865/RIDEP55.2.14

Cantor Parra, L. S., \& Clavijo Benavides, M. P. (2020). Adaptación y validación de la escala de afectividad positiva y negativa (PANAS) al contexto colombiano, en una muestra de madres solteras en la ciudad de Bogotá, Colombia (Tesis de grado). Fundación Universitaria Los libertadores, Bogotá, Colombia. https://hdl.handle.net/11371/2912

Cantril, H. (1965). The pattern of human concern. Rutgers University Press.

Casas, F., Fernández-Artamendi, S., Montserrat, C., Bravo, A., Bertrán, I., \& Del Valle, J. F. (2013). El bienestar 
subjetivo en la adolescencia: Estudio comparativo de dos Comunidades Autónomas en España. Anales de Psicología, 29(1), 148-158. https://dx.doi.org/10.6018/ analesps.29.1.145281

Castellá Sarriera, J., Saforcada, E., Tonon, G., Rodríguez de La Vega, L., Mozobancyk, S., \& Maria Bedin, L. (2012). Bienestar subjetivo de los adolescentes: Un estudio comparativo entre Argentina y Brasil. Psychosocial Intervention, 21(3), 273-280. https://doi.org/10.5093/ in $2012 \mathrm{a} 24$

Caycho, T. (2017). Importancia del análisis de invarianza factorial en estudios comparativos en Ciencias de la Salud. Revista Cubana de Educación Médica Superior, 31(2), 1-3. https://www.medigraphic.com/pdfs/educa cion/cem-2017/cem172d.pdf

Checa, I., Perales, J., \& Espejo, B. (2019). Measurement invariance of the Satisfaction with Life Scale by gender, age, marital status and educational level. Quality of Life Research, 28(4), 963-968. https://doi.org/10.1007/ s11136-018-2066-2

Chen, F. F., Sousa, K. H., \& West, S. G. (2005). Teacher's corner: Testing measurement invariance of second-order factor models. Structural Equation Modeling: A Multidisciplinary Journal, 12(3), 471-492. https://doi. org/10.1207/s15328007sem1203_7

Cheung, G. W., \& Rensvold, R. B. (2002). Evaluating goodness-of-fit indexes for testing measurement invariance. Structural Equation Modeling: A Multidisciplinary Journal, 9(2), 233-255. https://doi.org/10.1207/ S15328007SEM0902 5

Cuadra, H., \& Florenzano, R. (2003). El bienestar subjetivo: Haciaunapsicologíapositiva. RevistadePsicología, 12(1), 83-96. https://doi.org/10.5354/0719-0581.2003.17380

Determann, D., Lambooij, M., Steyerberg, E., Bekker-Grob, E., \& Ardine de Wit, G. (2017). Impact of survey administration mode on the results of a health-related discrete choice experiment: Online and paper comparison. Value in Health, 20(7), 953-960. https://doi.org/10.1016/j. jval.2017.02.007

Diener, E. D., Emmons, R. A., Larsen, R. J., \& Griffin, S. (1985). The Satisfaction with Life Scale. Journal of Personality Assessment, 49(1), 71-75. https://doi. org/10.1207/s15327752jpa4901_13

Diener, E., Lucas, R. E., \& Oishi, S. (2018). Advances and open questions in the science of subjective well-being. Collabra: Psychology, 4(1), 1-78. https://doi.org/10.1525 /collabra.115

Dimitrov, D. M. (2010). Testing for factorial invariance in the context of construct validation. Measurement and Evaluation in Counseling and Development, 43(2), 121149. https://doi.org/10.1177/0748175610373459

Ferrando, P. J., \& Anguiano-Carrasco, C. (2010). El análisis factorial como técnica de investigación en psicología. Papeles del Psicólogo, 31(1), 18-33. http://www.pape lesdelpsicologo.es/pdf/1793.pdf

Flores-Kanter, P. E., Garrido, L. E., Moretti, L. S., \& Medrano, L. A. (2021). A modern network approach to revisiting the Positive and Negative Affective Schedule (PANAS) construct validity. Journal of Clinical Psychology, 77(10), 2370-2404. https://doi.org/10.1002/jclp.23191

Flores-Kanter, P. E., \& Medrano, L. A. (2018). Comparación de dos versiones reducidas de la Escala PANAS: Análisis factoriales en una muestra argentina. Revista Iberoamericana de Diagnóstico y Evaluación e Avaliação Psicológica, 49(4), 37-46. https://doi.org/10.21865/ RIDEP49.4.03

Fornell, C., \& Larcker, D. F. (1981). Evaluating structural equation models with unobservable variables and measurement error. Journal of Marketing Research, 18(1), 39-50. https://doi.org/10.1177/002224378101800104

Furr, R. M. (2018). Psychometrics: An introduction (3. $\left.{ }^{\mathrm{a}} \mathrm{ed}.\right)$. Sage. 
Gómez, V., Villegas de Posada, C., Barrera, F., \& Cruz, J. E. (2007). Factores predictores de bienestar subjetivo en una muestra colombiana. Revista Latinoamericana de Psicología, 39(2), 311-325. https://www.redalyc.org/ pdf/805/80539208.pdf

Gutiérrez-Carmona, A., \& Urzúa M., A. (2019). ¿Los valores culturales afectan el bienestar humano? Evidencias desde los reportes de investigación. Universitas Psychologica, 18(1), 1-12. https://doi.org/10.11144/Javeriana.upsy181.vcab

Harding, S., Morris, R., Gunnell, D., Ford, T., Hollingworth, W., Tilling, K., Evans, R., Bell, S., Grey, J., Brockman, R., Campbell, R., Araya, R., Murphy, S., \& Kidger, J. (2019). Is teachers' mental health and wellbeing associated with students' mental health and wellbeing? Journal of Affective Disorders, 242(19), 180-187. https://doi.org /10.1016/j.jad.2018.08.080

Hassan Butt, T., Abid, G., Arya, B., \& Farooqi, S. (2020). Employee energy and subjective well-being: a moderated mediation model. The Service Industries Journal, 40(1-2), 133-157. https://doi.org/10.1080/02642069.2018.1563072

Hu, L., \& Bentler, P. M. (1999). Cutoff criteria for fit indexes in covariance structure analysis: Conventional criteria versus new alternatives. Structural Equation Modeling: A Multidisciplinary Journal, 6(1), 1-55. https://doi. org/10.1080/10705519909540118

Keyes, C., Shmotkin, D., \& Ryff, C. (2002). Optimizing well-being: the empirical encounter of two traditions. JournalofPersonalityandSocialPsychology, 82(6),10071022. https://doi.org/10.1037//0022-3514.82.6.1007

Kushlev, K., Drummond, D. M., \& Diener, E. (2020). Subjective Well-Being and Health Behaviors in 2.5 Million Americans. Applied Psychology: Health and Well-Being, 12(1), 166-187. https://doi.org/10.1111/ aphw.12178

Lapuente, L., Dominguez-Lara, S., Flores-Kanter, P. E., \& Medrano, L. A. (2018). Estructura del bienestar subjetivo mediante análisis bifactor: ¿Unidimensional $o$ multidimensional? AvaliaçãoPsicológica, 17(2),252-259. https://doi.org/10.15689/ap.2018.1702.14521.11

Levine, G. N., Cohen, B. E., Commodore-Mensah, Y., Fleury, J., Huffman, J. C., Khalid, U., Labarthe, D. R., Lavretsky, H., Michos, E. D., Spatz, E. S., Kubzansky, L. D., \& American Heart Association Council on Clinical Cardiology; Council on Arteriosclerosis, Thrombosis and Vascular Biology; Council on Cardiovascular and Stroke Nursing; and Council on Lifestyle and Cardiometabolic Health. (2021). Psychological health, well-being, and the mind-heart-body connection: A scientific statement from the American Heart Association. Circulation, 143(10), e763-e783. https://doi.org/10.1161/CIR.000000000000 0947

McCullough, M. E., Emmons, R. A., \& Tsang, J. A. (2002). The grateful disposition: A conceptual and empirical topography. Journal of Personality and Social Psychology, 82(1), 112-127. https://doi. org/10.1037/0022-3514.82.1.112

Medrano, L. A., Flores-Kanter, P. E., Trógolo, M., Curarello, A., \& González, J. (2015). Adaptación de la Escala de Afecto Positivo y Negativo (PANAS) para la población de Estudiantes Universitarios de Córdoba. Anuario de Investigaciones de la Facultad de Psicología, 2(1), 22-36. https://revistas.unc.edu.ar/index.php/aifp/article/down load/12503/12773

Moksnes, U. K., Løhre, A., Byrne, D. G., \& Haugan, G. (2014). Satisfaction with life scale in adolescents: Evaluation of factor structure and gender invariance in a Norwegian sample. Social Indicators Research, 118(2), 657-671. https://doi.org/10.1007/s11205-013-0451-3

Montero y López Lena, M. E. (1998). Soledad: Desarrollo y validación de un inventario multifacético para su medición (Tesis doctoral). Universidad Nacional Autónoma de México, México. http://132.248.9.195/pdbis/269524/ Index.html

Moral de la Rubia, J. (2011). La Escala de Afecto Positivo y Negativo (PANAS) en parejas casadas mexicanas. 
Ciencia Ergo-Sum, 18(2), 117-125. https://cienciaergo sum.uaemex.mx/article/view/7409

Moyano Díaz, E., \& Ramos Alvarado, N. (2007). Bienestar subjetivo: Midiendo satisfacción vital, felicidad y salud en población chilena de la Región Maule. Universum (Talca), 22(2), 177-193. https://doi.org/10.4067/ S0718-23762007000200012

Nima, A. A., Cloninger, K. M., Persson, B. N., Sikström, S., $\&$ Garcia, D. (2020). Validation of subjective well-being measures using Item Response Theory. Frontiers in Psychology, 10, 3036. https://doi.org/10.3389/ fpsyg.2019.03036

Padrós Blázquez, F., Gutiérrez Hernández, C. Y., \& Medina Calvillo, M. A. (2015). Propiedades psicométricas de la Escala de Satisfacción con la Vida (SWLS) de Diener en población de Michoacán (México). Avances en Psicología Latinoamericana, 33(02), 223-232. https:// doi.org/10.12804/ap133.02.2015.04

Pavot, W. (2018). The cornerstone of research on subjective well-being: Valid assessment methodology. En E. Diener, S. Oishi y L. Tay (Eds.), Handbook of Well-Being. DEF Publishers. https://www.nobascholar.com/chapters/15/ download.pdf

Pedrosa, I., Celis-Atenas, K., Suárez-Álvarez, J., GarcíaCueto, E., \& Muñiz, J. (2015). Cuestionario para la evaluación del optimismo: Fiabilidad y evidencias de validez. Terapia Psicológica, 33(2), 127-138. https://doi. org/10.4067/S0718-48082015000200007

Samejima, F. (1969). Estimation of latent ability using a response pattern of graded scores. Psychometrika Monographs, 34(Supl. 17). https://doi.org/10.1002/j.2333-8504.1968.tb00153.x

Simms, L. J. (2008). Classical and modern methods of psychological scale construction. Social and Personality Psychology Compass, 2(1), 414-433. https://doi. org/10.1111/j.1751-9004.2007.00044.x
Sociedad Mexicana de Psicología. (2010). Código ético del psicólogo (5. ${ }^{\mathrm{a}}$ ed.). Trillas.

Stöber, J., \& Joormann, J. (2001). A short form of the Worry Domains Questionnaire: Construction and factorial validation. Personality and Individual Differences, 31(4), 591-598. https://doi.org/10.1016/ S0191-8869(00)00163-X

Temiz, G. (2020). The relationship between mothers' psychological well-being and their attitudes towards their children. Psychology Research, 10(1), 1-9. https://doi. org/10.17265/2159-5542/2020.01.001

Tomás, J. M., Gutiérrez, M., Sancho, P., \& Romero, I. (2015). Measurement invariance of the Satisfaction with Life Scale (SWLS) by gender and age in Angola. Personality and Individual Differences, 85, 182-186. https://doi.org/10.1016/j. paid.2015.05.008

Van de Schoot, R., Lugtig, P., \& Hox, J. (2012). A checklist for testing measurement invariance. European Journal of Developmental Psychology, 9(4), 486-492. https://doi.or $\mathrm{g} / 10.1080 / 17405629.2012 .686740$

Vandenberg, R., \& Lance, C. (2000). A review and synthesis of the measurement invariance literature: Suggestions, practices, and recommendations for organizational research. Organizational Research Methods, 3(1), 4-70. https://doi.org/10.1177/109442810031002

Vinaccia Alpi, E., Parada, N., Quiceno, J. M., Riveros Munévar, F., \& Vera Maldonado, L. A. (2019). Escala de satisfacción con la vida (SWLS): Análisis de validez, confiabilidad y baremos para estudiantes universitarios de Bogotá. Psicogente, 22(42), 1-20. https://doi. org/10.17081/psico.22.42.3468

Watson, D., Clark, L.A., \& Tellegen, A. (1988). Development and validation of brief measures of positive and negative affect: The PANAS scales. Journal of Personality and Social Psychology, 54(6), 1063-1070. https://doi. org/10.1037//0022-3514.54.6.1063 